\title{
Linguistic Truth-Valued Intuitionistic Fuzzy Propositional Logic Based on LIA
}

\author{
Li Zou ${ }^{1}$ Shuiting $\mathrm{Wu}^{2}$ Zhengjiang $\mathrm{Wu}^{2}$ Yang $\mathrm{Xu}{ }^{2}$ \\ ${ }^{1}$ School of Computer and Information Technology, Liaoning Normal University, Dalian 116029, P.R.China \\ ${ }^{2}$ Intelligent Control Development Center, Southwest Jiaotong University, Chengdu 610031, P.R.China
}

\begin{abstract}
Truth degree and falsity degree of intuitionistic fuzzy proposition are two truth values with linguistic hedge. In this paper, using the framework of linguistic truth-valued propositional logic based on lattice implication algebra, a kind of linguistic truth-valued intuitionistic fuzzy propositional logic is constructed. Some logic properties regarding reasoning are then obtained. Especially, the implication operation of linguistic truth-valued intuitionistic fuzzy propositional logic can be deduced from four times implication of their truth values. Therefore, we can use more information in the process of reasoning and eventually improve the precision of reasoning.
\end{abstract}

Keywords: Lattice-valued logic, Linguistic truthvalued intuitionistic fuzzy logic, Approximate reasoning

\section{Introduction}

In the real world, people usually do judgement in a natural language with some uncertain words. In 1970s, Zadeh introduced and developed the theory of approximate reasoning based on the notions of a linguistic variable and fuzzy logic. The truth values of a fuzzy proposition are linguistic, e.g., of the form "true", "very true", "possible false", etc [1]-[2]. Therefor, truth values of proposition are often not exactly true or false, but accompany with linguistic hedges [4], such as absolute, highly, very, quite, exactly, almost, rather, somewhat, slightly and so on. These linguistic hedges can strengthen or weaken the degree of truth value. In recent years, some researchers have paid their attention to linguistic hedges. Ho proposed an algebraic model, Hedge Algebra, for dealing with linguistic information [6][7]. Turksen studied the formalization and inference of descriptive words, substantive words and declarative sentence [8]-[9]. Huynh[5] proposed a new model for parametric representation of linguistic truth-values[10]-[11].

$\mathrm{Xu}$ et.al. did some research on characterizing the set of linguistic values by a lattice-valued algebraic structure and investigate the corresponding logic systems with linguistic truth-value based on LIA [12]-[13]. From the point of lattice-valued logic system view[15]-[16], linguistic truth-values can be put into the lattice implication algebra(LIA)[17][18]. Zou [19] proposed a framework of linguistic truth-valued propositional logic and developed the reasoning method of six-element linguistic truthvalued logic system.

Sometimes, we analysis an event which has both certainty and uncertainty characteristic or has both obverse and inverse demonstration. Therefore, a proposition has two truth values: truth degree and falsity degree. From the view of intuitionistic fuzzy set introduced by K.Atanassov, the true value of a fuzzy proposition $p$ are juxtaposed two two real number $(\mu(p), \nu(p))$ on the closed interval $[0,1]$ with the following constraint:

$$
\mu(p)+\nu(p) \leq 1
$$

In [3] the evaluation function $V$ was defined over a set of propositions $S$ in such a way that

$$
V(p)=<\mu(p), \nu(p)>.
$$

Hence the function $V: S \rightarrow[0,1] \times[0,1]$ gives the truth and falsity degrees of all propositions in $S$. which represents its truth degree and its falsity degree [4].

With above work,we will put the linguistic truth-values into intuitionistic fuzzy logic. The truth values of the intuitionistic fuzzy logic are linguistic truth-values instead of number. Then we discuss the properties of linguistic truth-valued reasoning in intuitionistic fuzzy logic. 


\section{Framework of linguistic truth-valued logic}

In this section we briefly review the notion of linguistic truth-valued lattice implication algebra and its main properties.

Definition 1[14] Let $(L, \vee, \wedge, I, O, I)$ be a bounded lattice with universal boundaries $O$ (the least element) and $I$ (the greatest element) respectively, and " " be an order-reversing involution. For any $x, y, z \in L$, if mapping $\rightarrow: L \times L \rightarrow L$ satisfies:

$\left(I_{1}\right) x \rightarrow(y \rightarrow z)=y \rightarrow(x \rightarrow z)$,

$\left(I_{2}\right) x \rightarrow x=I$,

$\left(I_{3}\right) x \rightarrow y=y^{\prime} \rightarrow x^{\prime}$

$\left(I_{4}\right)$ if $x \rightarrow y=y \rightarrow x=I$, then $x=y$,

$\left(I_{5}\right)(x \rightarrow y) \rightarrow y=(y \rightarrow x) \rightarrow x$,

$\left(I_{6}\right)(x \vee y) \rightarrow z=(x \rightarrow z) \wedge(y \rightarrow z)$,

$\left(I_{7}\right)(x \wedge y) \rightarrow z=(x \rightarrow z) \vee(y \rightarrow z)$,

then $(L, \vee, \wedge, \prime, \rightarrow, O, I)$ is called a lattice implication algebra.

Definition 2 Denote $C=\left\{c_{i} \mid c_{1}=\operatorname{false}(F)\right.$, $\left.c_{2}=\operatorname{true}(T), i=1,2\right\}$, which is called as the set of meta truth values. The lattice implication algebra (of course a Booleaan algebra) defined on the set of meta truth values is called a meta linguistic truth-valued lattice implication algebra, where $F<T$, the operation "'" is defined as: $T^{\prime}=F$ and $F^{\prime}=T$, the operation " $\rightarrow$ " is defined as:

$$
\begin{aligned}
& \rightarrow: C \times C \rightarrow C, \\
& x \rightarrow y=x^{\prime} \vee y .
\end{aligned}
$$

Definition 3 Denote $H=\left\{h_{i} \mid i=0 \ldots \ldots n, \mathrm{n}\right.$ is an even number $\}$, which is called linguistic hedges set. Here we assume they are a chain, $h_{i}<h_{j}$, if $i<j$. For $0 \leq i, j \leq n$, define:

$$
\begin{gathered}
h_{i} \vee h_{j}=h_{\max \{i, j\}} \\
h_{i} \wedge h_{j}=h_{\min \{i, j\}} \\
h_{i}^{\prime}=h_{n-i} \\
h_{i} \rightarrow h_{j}=h_{\min \{n, n-i+j\}}
\end{gathered}
$$

then $\left(H, \vee, \wedge,{ }^{\prime}, \rightarrow\right)$ is a LIA.

Let $V$ be a linguistic truth values set, every linguistic truth value $v \in V$ is composed of a linguistic hedge operator $h$ and a basic word $c$, i.e. $V=H \times$ $C$ where the linguistic hedge operator set $H$ is a totally ordered and finite set.

According to the characteristic of lattice implication algebra, we can construct a new lattice implication algebra using the product of some lattice implication algebras.

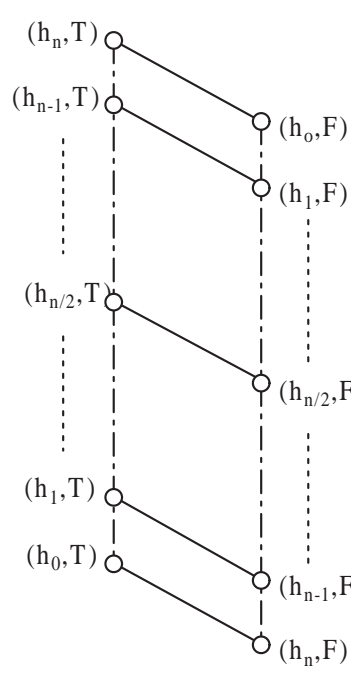

Fig. 1: Hasse Diagram of $L$.

Definition 4 Let $V=H \times C$, denote $L=(V, \vee$, $\wedge$, ', $\rightarrow$ ), its operation " $\vee$ " and " $\wedge$ " are shown in the Hasse diagram of $L$ defined in $1,\left(h_{i}, T\right)^{\prime}=\left(h_{i}, F\right)$, $\left(h_{i}, F\right)^{\prime}=\left(h_{i}, T\right)$ and its operations " $\rightarrow$ " defined as follows:

$$
\left\{\begin{aligned}
\left(h_{i}, T\right) \rightarrow\left(h_{j}, F\right) & =\left(h_{\max \{0, i+j-n\}}, F\right) \\
\left(h_{i}, F\right) \rightarrow\left(h_{j}, T\right) & =\left(h_{\min \{n, i+j\}}, T\right) \\
\left(h_{i}, T\right) \rightarrow\left(h_{j}, T\right) & =\left(h_{\min \{n, n-i+j\}}, T\right) \\
\left(h_{i}, F\right) \rightarrow\left(h_{j}, F\right) & =\left(h_{\min \{n, n-j+i\}}, T\right)
\end{aligned}\right.
$$

Then $L=\left(V, \vee, \wedge,{ }^{\prime}, \rightarrow\right)$ (in short $L$ ) is a lattice implication algebra.

Proposition 1 For any formula $p, q$ of LTVP, $\left(h_{i}, c_{j}\right) \in L$,

$(1) \sim\left(\left(h_{i}, c_{j}\right) p\right)=\left(h_{i}, c_{j}^{\prime}\right) p$

$(2) \sim\left(\left(h_{i}, c_{j}\right) p\right)=\left(h_{i}, c_{j}\right)(\sim p)$

$(3)\left(h_{i}, c_{i}\right)(p \vee q)=\left(h_{i}, c_{i}\right) p \vee\left(h_{i}, c_{i}\right) q$

(4) $\left(h_{i}, c_{i}\right)(p \wedge q)=\left(h_{i}, c_{i}\right) p \wedge\left(h_{i}, c_{i}\right) q$

Proposition 2 For any $\left(h_{i}, c_{j}\right) \in L$,

(1) $\left(h_{n}, F\right) \rightarrow\left(h_{i}, c_{j}\right)=\left(h_{n}, T\right)$,

(2) $\left(h_{n}, T\right) \rightarrow\left(h_{i}, c_{j}\right)=\left(h_{i}, c_{j}\right)$,

(3) $\left(h_{i}, c_{j}\right) \rightarrow\left(h_{n}, F\right)=\left(h_{i}, c_{j}\right)^{\prime}$,

(4) $\left(h_{i}, c_{j}\right) \rightarrow\left(h_{n}, T\right)=\left(h_{i}, c_{j}\right)$.

\section{Linguistic truth-valued intu- itionistic fuzzy logic}

Since some kinds of truth and falsity are incomparable, we can choose the linguistic truth-values based on LIA as the truth-valued field of intuitionistic fuzzy logic. We denote the linguistic truth-valued intuitionistic fuzzy proposition by LTV-IFP. 
The symbols in LTV-IFP Logic system are

(1) The set of propositional variable: $X=$ $\{p, q, r, \ldots\}$;

(2) The set of constants: $L=\left\{\left(\left(h_{i}, T\right),\left(h_{j}, F\right)\right)\right\}$;

(3) Logical connectives: $\rightarrow$, ';

(4) Auxiliary symbols: ),(.

The set $F$ of formulae of LTV-IFP is the least set $Y$ satisfying the following conditions:

(1) $X \subseteq Y$;

(2) $L \subseteq Y$;

(3)If $p, q \in Y$,then $p^{\prime}$ and $p \rightarrow q \in Y$.

Note that from the viewpoint of universal algebra, LTV-IFP is the free algebra on $X$ w.r.t. the type $T=L \cup\left\{{ }^{\prime}, \rightarrow\right\}$, where $\alpha \in L$ is a 0 -ary operation.

According to the properties of lattice implication algebra, $L$ and LTV-IFP can be looked as algebras with the same type $T=L \cup\left\{{ }^{\prime}, \rightarrow\right\}$ and for any $p, q \in F$,

(1) $p \vee q=(p \rightarrow q) \rightarrow q$,

(2) $p \wedge q=\left(p^{\prime} \vee q^{\prime}\right)$.

Definition 5 A mapping

$$
v: L T V-I F P \rightarrow\left(\left(h_{i}, T\right),\left(h_{j}, F\right)\right),
$$

where $\left(\left(h_{i}, T\right),\left(h_{j}, F\right)\right) \in L \times L$, where $i+j \leq n$ is called a valuation of LTV-IFP, if it is a $T$ homomorphism. The conjunction, disjunction and implication are shown as follows:

Let $G, H \in L T V-I F P, v(G)=\left(\left(h_{i}, T\right)\right.$, $\left.\left(h_{j}, F\right)\right), v(G)=\left(\left(h_{m}, T\right),\left(h_{l}, F\right)\right)$,

1.v $(G \vee H)=\left(\left(h_{i}, T\right) \vee\left(h_{m}, T\right),\left(h_{j}, F\right) \wedge\left(h_{l}, F\right)\right)$;

2.v $(G \wedge H)=\left(\left(h_{i}, T\right) \wedge\left(h_{m}, T\right),\left(h_{j}, F\right) \vee\left(h_{l}, F\right)\right)$.

3. $v(G \rightarrow H)=v(G) \rightarrow v(H)=\left(\left(\left(h_{i}, T\right) \rightarrow\right.\right.$

$\left.\left(h_{m}, T\right)\right) \wedge\left(\left(h_{j}, F\right) \rightarrow\left(h_{m}, T\right) \wedge\left(\left(h_{j}, F\right) \rightarrow\right.\right.$

$\left.\left.\left.\left(h_{l}, F\right)\right)\right),\left(h_{i}, T\right) \rightarrow\left(h_{l}, F\right)\right)$;

Note that, for 1 and 2 they satisfy the valuation conditions of LTV-IFP obviously.

For 3, we get

$v(G \rightarrow H)$

$=v(G) \rightarrow v(H)$

$=\left(\left(\left(h_{i}, T\right) \rightarrow\left(h_{m}, T\right)\right) \wedge\left(\left(h_{j}, F\right) \rightarrow\right.\right.$

$\left.\left.\left(h_{m}, T\right) \wedge\left(\left(h_{j}, F\right) \rightarrow\left(h_{l}, F\right)\right)\right),\left(h_{i}, T\right) \rightarrow\left(h_{l}, F\right)\right)$

$=\left(\left(h_{\min \{n, n-i+m\}}, T\right) \wedge\left(h_{\min \{n, j+m\}}, T\right) \wedge\right.$

$\left.\left(h_{\min \{n, n-l+j\}}, T\right),\left(h_{\max \{0, i+l-n\}}, F\right)\right)$

For the truth degree of $G \rightarrow H$ there are four cases, the subscripts are $n, n-i+m, j+m, n-l+j$ respectively. For the falsity degree of $G \rightarrow H$, the subscript is $i+l-n$. We can prove that the sum is always equal to or less than $n$.

Hence the definitions of conjunction, disjunction and implication of LTV-IFP are rational.

Corollary 1 Let

$$
v: L T V-I F P \rightarrow\left(\left(h_{i}, T\right),\left(h_{j}, F\right)\right)
$$

be a mapping, then $v$ is a valuation of LTV-IFP if and only if it satisfies

(1) $v\left(\left(h_{\alpha}, T\right),\left(h_{\beta}, F\right)\right)=\left(\left(h_{\alpha}, T\right),\left(h_{\beta}, F\right)\right)$, for any

$\left(\left(h_{\alpha}, T\right),\left(h_{\beta}, F\right)\right) \in L$ and $\alpha+\beta \leq 1$;

$(2) v\left(p^{\prime}\right)=(v(p))^{\prime}$ for any $p \in F$;

$(3) v(p \rightarrow q)=v(p) \rightarrow v(q)$ for any $p, q \in F$.

Definition 6 Let $p$ be a symbol of an LTV-IFP atom, and $v(p)=\left(\left(h_{i}, T\right),\left(h_{j}, F\right)\right)$, where $i+j \leq n$. LTV-IFP atom is the fundamental element of LTVIFP.

Definition 7 Well-formed formula of LTV-IFP or formula for short are defined recursively as follows:

(1) LTV-IFP atom is a formula;

(2) If $G, H$ are LTV-IFP formulae, then $\sim G$, $(G \vee H),(G \wedge H),((G \rightarrow H)$ and $(G \rightarrow H)$ are formulae;

(3) No expression is a formula unless it is compelled to be one by (1) and (2).

There are five types of "nagation" in LTV-IFP formally, let $v(p)=\left(\left(h_{i}, T\right),\left(h_{j}, F\right)\right)$ :

$1 . v\left(p^{\prime}\right)=\left(\left(h_{i}, F\right),\left(h_{j}, T\right)\right)$;

2.v $\left(p^{\prime}\right)=\left(\left(h_{n-i}, T\right),\left(h_{n-j}, F\right)\right)$;

3.v $\left(p^{\prime}\right)=\left(\left(h_{j}, T\right),\left(h_{i}, F\right)\right)$;

4.v $\left(p^{\prime}\right)=\left(\left(h_{n-i}, F\right),\left(h_{n-j}, T\right)\right)$;

$5 . v\left(p^{\prime}\right)=\left(\left(h_{j}, F\right),\left(h_{i}, T\right)\right)$.

Considering the restriction of valuation of LTV-IFP and people's intuition, the second and the third are both better than others. We would like to choose the third negation to discuss.

Some intuitionistic linguistic truth-valued properties hold as follows:

Theorem 1 For any $\left(\left(h_{i}, T\right),\left(h_{j}, F\right)\right) \in L$, where $i+j \leq 1$,

(1) $\left(\left(h_{0}, T\right),\left(h_{n}, F\right)\right) \rightarrow\left(\left(h_{i}, T\right),\left(h_{j}, F\right)\right)=$ $\left(\left(h_{n}, T\right),\left(h_{0}, F\right)\right)$,

(2) $\left(\left(h_{n}, T\right),\left(h_{0}, F\right)\right) \rightarrow\left(\left(h_{i}, T\right),\left(h_{j}, F\right)\right)=$ $\left(\left(h_{i}, T\right),\left(h_{j}, F\right)\right)$

(3) $\left(\left(h_{i}, T\right),\left(h_{j}, F\right)\right) \rightarrow\left(\left(h_{0}, T\right),\left(h_{n}, F\right)\right)=$ $\left(\left(h_{j}, T\right),\left(h_{i}, F\right)\right)$

(4) $\left(\left(h_{i}, T\right),\left(h_{j}, F\right)\right) \rightarrow\left(\left(h_{n}, T\right),\left(h_{0}, F\right)\right)=$ $\left(\left(h_{n}, T\right),\left(h_{0}, F\right)\right)$.

Proof. For (1),

$\left(\left(h_{0}, T\right),\left(h_{n}, F\right)\right) \rightarrow\left(\left(h_{i}, T\right),\left(h_{j}, F\right)\right)$

$=\left(\left(\left(h_{0}, T\right) \rightarrow\left(h_{i}, T\right)\right) \wedge\left(\left(h_{n}, F\right) \rightarrow\left(h_{i}, T\right)\right) \wedge\right.$

$\left.\left.\left(\left(h_{n}, F\right) \rightarrow\left(h_{j}, F\right)\right)\right),\left(h_{0}, T\right) \rightarrow\left(h_{j}, F\right)\right)$

$=\left(\left(h_{\min \{n, n-0+i\}}, T\right) \wedge\left(h_{\min \{n, n+i\}}, T\right) \wedge\right.$

$\left.\left(h_{\min \{n, n-j+n\}}, T\right),\left(h_{\max \{0,0+j-n\}}, F\right)\right)$

$=\left(\left(h_{n}, T\right) \wedge\left(h_{n}, T\right) \wedge\left(h_{n}, T\right),\left(h_{0}, F\right)\right)$

$=\left(\left(h_{n}, T\right),\left(h_{0}, F\right)\right)$.

For $(2)$, since $i+j \leq 1$

$\left(\left(h_{n}, T\right),\left(h_{0}, F\right)\right) \rightarrow\left(\left(h_{i}, T\right),\left(h_{j}, F\right)\right)$

$=\left(\left(\left(h_{n}, T\right) \rightarrow\left(h_{i}, T\right)\right) \wedge\left(\left(h_{0}, F\right) \rightarrow\left(h_{i}, T\right) \wedge\right.\right.$ 


$$
\begin{aligned}
& \left.\left.\left(\left(h_{0}, F\right) \rightarrow\left(h_{j}, F\right)\right)\right),\left(h_{n}, T\right) \rightarrow\left(h_{j}, F\right)\right) \\
= & \left(\left(h_{\min \{n, n-n+i\}}, T\right) \wedge\left(h_{\min \{n, 0+i\}}, T\right) \wedge\right. \\
& \left.\left(h_{\min \{n, n-j+0\}}, T\right),\left(h_{\max \{0, n+j-n\}}, F\right)\right) \\
= & \left(\left(h_{i}, T\right) \wedge\left(h_{i}, T\right) \wedge\left(h_{n-j}, T\right),\left(h_{j}, F\right)\right) \\
= & \left(\left(h_{i}, T\right),\left(h_{j}, F\right)\right) .
\end{aligned}
$$

(3) and (4) can be proved analogously.

Corollary 2 For any $\left(\left(h_{i}, T\right),\left(h_{j}, F\right)\right) \in L$, where $i+j \leq 1$,

(1) $\left(\left(h_{0}, T\right),\left(h_{n}, F\right)\right) \rightarrow\left(\left(h_{0}, T\right),\left(h_{n}, F\right)\right)=$ $\left(\left(h_{n}, T\right),\left(h_{0}, F\right)\right)$

(2) $\left(\left(h_{n}, T\right),\left(h_{0}, F\right)\right) \rightarrow\left(\left(h_{n}, T\right),\left(h_{0}, F\right)\right)=$ $\left(\left(h_{n}, T\right),\left(h_{0}, F\right)\right)$

(3) $\left(\left(h_{n}, T\right),\left(h_{0}, F\right)\right) \rightarrow\left(\left(h_{0}, T\right),\left(h_{n}, F\right)\right)=$ $\left(\left(h_{0}, T\right),\left(h_{n}, F\right)\right)$

(4) $\left(\left(h_{0}, T\right),\left(h_{n}, F\right)\right) \rightarrow\left(\left(h_{n}, T\right),\left(h_{0}, F\right)\right)=$ $\left(\left(h_{n}, T\right),\left(h_{0}, F\right)\right)$.

Definition 8 For any $\left(\left(h_{i}, T\right),\left(h_{j}, F\right)\right)$, $\left(\left(h_{m}, T\right),\left(h_{l}, F\right)\right) \in L$, where $i+j \leq 1$ and $m+l \leq 1, \quad\left(\left(h_{i}, T\right),\left(h_{j}, F\right)\right)$ is said to truer than $\left(\left(h_{m}, T\right),\left(h_{l}, F\right)\right)$ if and only if $\left(h_{i}, T\right) \geq\left(h_{m}, T\right)$ and $\left(h_{j}, F\right)<\left(h_{l}, F\right)$ or $\left(h_{i}, T\right)>\left(h_{m}, T\right)$ and $\left(h_{j}, F\right) \leq\left(h_{l}, F\right)$, denoted by $\left(\left(h_{i}, T\right),\left(h_{j}, F\right)\right) \geq\left(\left(h_{m}, T\right),\left(h_{l}, F\right)\right)$.

\section{Theorem 2 If}

$$
\left(\left(h_{i}, T\right),\left(h_{j}, F\right)\right) \geq\left(\left(h_{m}, T\right),\left(h_{l}, F\right)\right),
$$

then

$$
\begin{aligned}
& \left(\left(h_{i}, T\right),\left(h_{j}, F\right)\right) \rightarrow\left(\left(h_{0}, T\right),\left(h_{n}, F\right)\right) \leq \\
& \left(\left(h_{m}, T\right),\left(h_{l}, F\right)\right) \rightarrow\left(\left(h_{0}, T\right),\left(h_{n}, F\right)\right) .
\end{aligned}
$$

Proof. From theorem 1, we get

$$
\begin{aligned}
& \left(\left(h_{i}, T\right),\left(h_{j}, F\right)\right) \rightarrow\left(\left(h_{0}, T\right),\left(h_{n}, F\right)\right) \\
= & \left(\left(h_{j}, T\right),\left(h_{i}, F\right)\right) ; \\
& \quad\left(\left(h_{m}, T\right),\left(h_{l}, F\right)\right) \rightarrow\left(\left(h_{0}, T\right),\left(h_{n}, F\right)\right) \\
= & \left(\left(h_{l}, T\right),\left(h_{m}, F\right)\right) .
\end{aligned}
$$

Since $\left(\left(h_{i}, T\right),\left(h_{j}, F\right)\right) \geq\left(\left(h_{m}, T\right),\left(h_{l}, F\right)\right)$, and the linguistic hedge set $H=h_{i} \mid i=1,2 \ldots n$ is a chain, then we get

$$
\left(\left(h_{j}, T\right),\left(h_{i}, F\right)\right) \leq\left(\left(h_{l}, T\right),\left(h_{m}, F\right)\right) .
$$

Note that if the consequence is the most false then the truth degree of the implication will decrease while the truth degree of the premise increases. Conversely, while the truth degree of the premise decreases, the truth degree of the implication will increase. This property is consistent with the classical logic and people's intuition. Also, the linguistic truth-values based on LIA are special cases of intuitionistic linguistic truth-values. So the linguistic truth-valued intuitionistic logic is an extension of linguistic truth-valued logic.

Theorem 3 For any $\left(\left(h_{i}, T\right),\left(h_{j}, F\right)\right)$, $\left(h_{i}, T\right),\left(h_{l}, F\right),\left(\left(h_{m}, T\right),\left(h_{j}, F\right)\right) \in L$, $(1)\left(\left(h_{i}, T\right),\left(h_{j}, F\right)\right) \rightarrow\left(h_{i}, T\right),\left(h_{l}, F\right)$ $=\left(\left(h_{i+j}, T\right),\left(h_{0}, F\right)\right)$,

$(2)\left(\left(h_{i}, T\right),\left(h_{j}, F\right)\right) \rightarrow\left(\left(h_{m}, T\right),\left(h_{j}, F\right)\right)$

$=\left(\left(h_{j+m}, T\right),\left(h_{0}, F\right)\right)$,

Now when we do fuzzy inference in intuitionistic fuzzy logic system based on linguistic truthvalue, we must consider the fact that the proposition has the truth degree as well as the falsity degree. So more information is used in the reasoning process, which can improve the precision of reasoning and reduce the loss of information in a sense

\section{Conclusions}

We have found that some properties of latticevalued logic based on linguistic truth-valued are fit for researching linguistic truth-values. The result is consistent with people's intuition. The classical logic and linguistic truth-valued logic based on LIA are the special cases of this logic system.

The problem which has positive evidence and negative evidence at the same time can be dealt with by means of linguistic truth-value intuitionistic fuzzy logic. If a proposition has both credibility and incredibility, then the reasoning method proposed above can be used.

The further work is to build a reasoning model for linguistic truth-valued intuitionistic logic. We also hope this method can be applied into the fields of decision-making, evaluation, risk assessment and so on

\section{Acknowledgement}

This work is partially supported by National Nature Science Foundation of China (Grant No.60474022 and 60603047)and the Specialized Research Fund for the Doctoral Program of Higher Education of China under Grant No. 20060613007.

\section{References}

[1] L.A.Zadeh, The comcept of linguistic variable and its application to approximate reasoning (I), Information Sciences, 8:199-249, 1975.

[2] L.A.Zadeh, The comcept of linguistic variable and its application to approximate reasoning (II), Information Sciences, 8:310-357, 1975.

[3] K.Atanassov, Elements of intuitionistic fuzzy logic. Part I, Fuzzy Set and Systems, 95:39-52, 1998. 
[4] F. Herrera, E.Herrera, Luis Martinez, A Fusion Approach for Managing Multi-granularity Linguistic Term Sets in Decision Making, Fuzzy Sets and Systems 114:43-58, 2000.

[5] V. N. Huynh, H. V. Nam, Ordered Structurebased Semantics of Linguistic Terms of Linguistic Variables and Approximate Reasoning, AIP Conference Proceedings on Computing Anticipatory Systems, CASYS'g9 Third Tinternational Conference, pp.98-116, 1999.

[6] N. C. Ho and W. Wechler, Hedge algebras: an algebraic approach to structure of sets of linguistic turth values, Fuzzy Sets and Systems, 35: 281-293, 1990.

[7] N. C. Ho and W. Wechler,Extended hedge algebras and their application to fuzzy logic, Fuzzy Sets and Systems, 52: 259-281, 1992.

[8] I.B. Turksen, Computing with descriptive and verisic words, Proc. of NAFIP'99, pp.13-17, 1999.

[9] I.B. Turksen, A.Kandel, and Y. Q. Zhang, Universal truth tables and normal forms, IEEE Trans. Fuzzy Systems, 6: 295-303, 1998.

[10] V. N. Huynh, T. B. Ho, and Y. Nakamori, A Parametric Representation of Linguistic Hedges in Zadeh's Fuzzy Logic, International Journal of Approximate Reasoning, 30: 203-223, 2002.

[11] C. H. Nguyen, V. N. Huynh, An algebraic approach to linguistic hedges in Zadeh's fuzzy logic, Fuzzy Set and Systems, 129: 229-254, 2002.

[12] Y. Xu, D. Ruan, K. Y. Qin and J. Liu, Latticevalued Logic, Springer Published, 2004.

[13] Y. Xu, D. Ruan, E. E. Kerre, J. Liu, $\alpha$-resolution principle based on lattice-valued propositional logic $\mathrm{LP}(\mathrm{X})$, Information Sciences, 130: 195-223, 2000.

[14] Y. Xu, D. Ruan, E.E. Kerre, J. Liu, $\alpha$ - resolution principle based on first-order latticevalued propositional logic $\mathrm{LF}(\mathrm{X})$, Information Sciences, 132: 195-223, 2001.

[15] Y. Xu, J. Liu, D. Ruan, and T.T. Lee,On the consistency of rule bases based on lattice-valued first-order logic LF(X), International Journal of Intelligent Systems, 21: 399-424, 2006.

[16] Z. Pei, Y. Xu, Lattice implication algebra model of a kind of linguistic terms and its in inference, Proc. of the 6th Internal FLINS Conferece, pp. 93-98, 2004.

[17] L. Zou, J. Ma, Y. Xu, A Framework of Linguistic Truth-valued Propositional Logic Based on Lattice Implication Algebra, Proceedings of 2006 IEEE International Conference on Granular Computing, pp. 574-577, 2006.
[18] L. Zou, P. Das, Y. Xu, D. Meng, Softresolutiion Method of Linguistic Hedges Lattice-valued First-order Logic. Proceedings of 8th International Joint Conference on Information Science, pp.136-139, 2005.

[19] L. Zou, X. Liu, Y. Xu, Resolution Method of Linguistic Truth-valued Propositional Logic, International Conference on Neural Networks and Brain, pp. 1996-2000, 2005. 